\title{
Use of Electronic Health (eHealth) Among Saudi Type 2 Diabetic Patients and Its Association With Their Diabetic Self-Management: A Cross- Sectional Study
}

Asmaa Abdel Nasser ${ }^{1}$, Razan M. Alzahrani ${ }^{2}$, Ahmed N. Ghandoura ${ }^{2}$, Intessar Sultan ${ }^{3}$

1. Medical Education, Faculty of Medicine, Suez Canal University, Ismailia, EGY 2. Medicine, Ibn Sina National College for Medical Studies, Jeddah, SAU 3. Internal Medicine, Ibn Sina National College for Medical Studies, Jeddah, SAU

Corresponding author: Asmaa Abdel Nasser,dr.asmaashraf@gmail.com

\section{Abstract}

\section{Background}

Type 2 diabetes mellitus (T2DM) is a prevalent, chronic, non-communicable disease that requires continuous multidisciplinary health care. Electronic health (eHealth) refers to "the transfer of health information resources and health care services using different electronic platforms.” This may have an effect on diabetes self-management (DSM).

\section{Objectives}

This study aimed to identify the use of eHealth among patients with T2DM as well as its association with DSM.

\section{Method}

An analytical cross-sectional study was conducted online using a newly adapted three-part questionnaire using Google Forms through different social media platforms. A total of 2,228 adult Saudi T2DM patients from different provinces were selected based on the non-probability voluntary response sampling technique. The survey included demographic, clinical, and eHealth data, and diabetic self-care management.

\section{Results}

The study results revealed an average DSM score of $5.2 / 10$, and $74.1 \%$ were receiving diabetes care at primary health care centers. Of these, $87.1 \%$ used eHealth, mainly through Google (55.7\%) and other social media (12.9\%), and were satisfied with the quality of health care (70.4\%). Moreover, $82 \%$ wanted to discuss the eHealth information with their physicians, but some (34.5\%) had no online access to them. eHealth dependency was $44.2 \%$ and was associated with a lower mean DSM (5.6 vs. $5.3 ; \mathrm{p}=0.000$ ) with significantly lower health care use ( 6.7 vs. $5.6 ; p=0.000$ ) and glucose management ( 4.7 vs. $4.0, p=0.000$ ) compared to the independent group. The DSM total score was a significant predictor of eHealth dependency (OR: 1.022; 95\%

Review began 03/09/2021 Review ended 03/12/2021 Published 03/14/2021

\section{() Copyright 2021}

Abdel Nasser et al. This is an open access article distributed under the terms of the Creative Commons Attribution License CC-BY 4.0., which permits unrestricted use, distribution, and reproduction in any medium, provided the original author and source are credited.
CI: $1.006-1.039 ; \mathrm{p}=0.007)$.

\section{Conclusion}

Most Saudi T2DM patients with an average DSM use different eHealth resources and are satisfied with their quality. Dependency to eHealth is significantly associated with lower DSM, especially for health care use and glucose management, a finding that could affect patient outcomes. Still, patients need to communicate with their physicians in person who should have different options for remote consultation, such as telemedicine, to support their patients.

Categories: Endocrinology/Diabetes/Metabolism, Family/General Practice, Healthcare Technology Keywords: electronic health, ehealth, type $2 \mathrm{dm}$, health care visits, self-care management

\section{Introduction}

Type 2 diabetes mellitus (T2DM) is a prevalent complex, chronic, non-communicable disease that requires continuous multidisciplinary health care. Ongoing diabetes self-management (DSM) education is crucial for preventing acute complications, reducing the risk of long-term complications, and improving patient outcomes [1]. Proper DSM support should follow the steps of evidence-based medicine and be initiated and monitored by skilled health professionals in a consistent manner. It should follow well-designed programs that address all patient aspects and challenges [2].

The term eHealth (electronic health) refers to "the transfer of health information resources and health care 
services using different electronic platforms" using the Internet [3]. It is currently an area of evolution with a possible impact on DSM. eHealth can support the exchange of medical information, improve communication, and provide patient education [4]. It shows an exceptional impact on diabetic patients who are in need of continued information and self-care, as well as frequent monitoring and regular follow-up visits $[5,6]$. Understanding the interaction between eHealth and health care utilization among patients with diabetes is of great importance for constructing evidence-based regulations for better health outcomes [7]. The interaction of eHealth with diabetes care has some positive and negative impacts. Researchers have reported an increasing number of diabetic patients using eHealth with potential benefits and risks of diabetes care [8-10]. eHealth can provide essential information before or after the hospital visit or direct patients to seek medical consultation [11]. eHealth risks include retrieving inaccurate, incomplete, and nonevidence-based information, especially if the users are of low health literacy levels or if there is a lack of direct communication with their health care providers [12-14]. The risks could also include spreading rumors or misinformation and endangering patients' privacy [15]. Still, there is a need for more research from different cultural and economic settings to outline the benefits and risks of using eHealth among diabetic patients [7].

Most (83.9\%) of the population with diabetes mellitus (DM) in the region are living in low- or middle-income countries. Saudi Arabia and Kuwait are among the countries with high diabetes prevalence (raw diabetes prevalence of $17.6 \%$ and $14.3 \%$, respectively). The countries with the largest number of adults with diabetes are Egypt (7.8 [3.8-9.0] million), Pakistan (7.0 [5.1-10.0] million), and Iran (4.6 [3.6-6.3] million) [16]. One study in Saudi from two large public university hospitals in Riyadh reported the use of eHealth by almost one-quarter of the T2DM patients and that eHealth information represented the second source of their selfcare data after consultation of their physicians [17]. Therefore, the aim of this study was to identify the use of eHealth among Saudi T2DM patients as well as its association with their DSM.

\section{Materials And Methods}

\section{Study context and design}

An analytical cross-sectional study was conducted by Ibn Sina National College for Medical Studies, Jeddah, Saudi Arabia, among Saudi T2DM patients. The study duration was six months (October 2019 to March 2020). The inclusion criteria included Saudi adults diagnosed with T2DM for at least one year up to five years. Patients with incomplete responses were excluded from the study. The sample size for the study was set at 2,200 participants, with a $95 \%$ confidence level and 2.75 error of precision.

\section{Data collection methods}

A newly adapted three-section online questionnaire to measure T2DM eHealth dependency was sent via Google form through different electronic platform applications to T2DM patients who were selected using the non-probability voluntary response sampling technique (Figure 1). The first section of the questionnaire included the demographic data of the participants (age, gender, and regional area). The second section addressed the use of eHealth, which consisted of 26 closed questions that were developed and modified in accordance with the research objectives. The third section collected data on diabetes duration, treatment, frequency of visits, awareness of diabetes care (questions about type and number of point-of-care lab tests during follow-up), concerns, and satisfaction. Then, eHealth methods and applications were used to follow up and assess its association with changes in the patients' diabetic care. Patients who agreed to replace the physician consultation with eHealth modalities were considered as the eHealth-dependent group. 


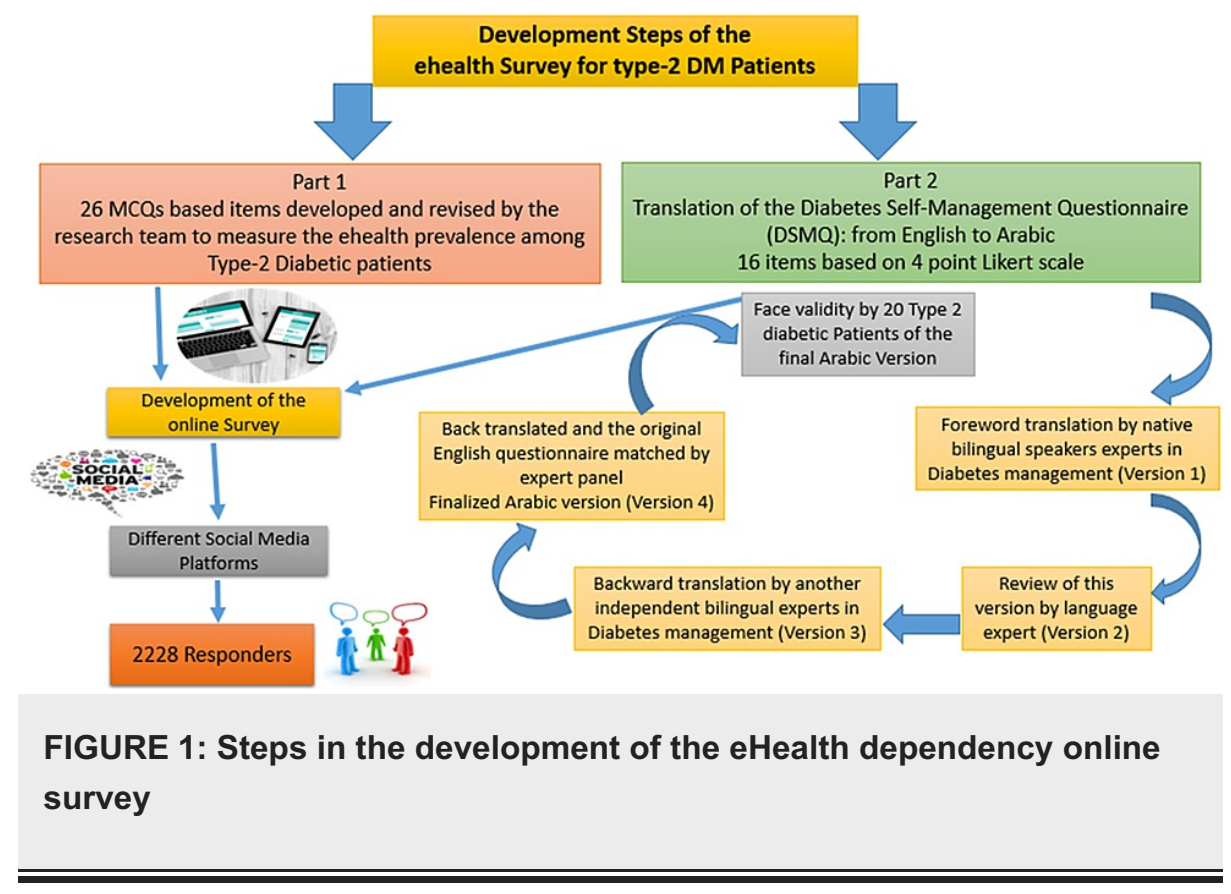

The participants were also asked to respond to an Arabic version of the Diabetes Self-Management Questionnaire (DSMQ). The DSMQ is a reliable and valid instrument for evaluating diabetes self-care behaviors in association with glycemic control [18]. The questionnaire was translated into Arabic using a standardized forward and backward translation procedure, as recommended by Bradley [19]. Participants were rated using the 16 items of the DSMQ, describing specific self-care behaviors according to their own diabetes control during the last one to five years. Rating is done based on a four-point Likert scale (from 0 "does not apply to me" to 3 "applies to me very much"; a neutral response option is not available in order to extract more specific results plus avoid of central tendency effect). The questionnaire contained 16 items: five for glucose management, four for dietary control, three for physical activity, three for health-care use, and one general question item. Of these 16 items, seven were formulated positively and nine were inversely formulated to calculate the total summation score. Then, each score was transformed into a score out of 10 (raw score/maximum score x10). Therefore, the higher the score, the better the self-management of diabetes.

\section{Ethical considerations}

Ethical approval for this study was obtained from the Ibn Sina National College Research and Ethics Committee in accordance with the Declaration of Helsinki for human studies [20]. During the online survey, the participants were informed about the purpose of the study and their right to refuse participation. Ethical conduct was maintained during data collection and throughout the research. Participation in the study was voluntary, and the confidentiality of the participants was maintained as the questionnaire was provided anonymously.

\section{Statistical analysis}

The collected data were coded and analyzed by a computer using a database software program, Statistical Package for Social Science (SPSS) Version 20 (IBM Corp., Armonk, NY, USA). Quantitative variables are expressed as numbers and percentages. The DSM individual and total scores were calculated out of 10, and their median results were used to construct Figure 1 using excel sheets and compare between the two groups eHealth dependency using the independent samples non-parametric Kruskal-Wallis U test. Logistic regression (binary regression) analysis was used to detect the significant predictors of eHealth dependency, and the exponential B with its $95 \%$ confidence interval (CI) was considered the odds ratio (OR). The results were considered statistically significant when the two-tailed p-value was less than 0.05 .

\section{Results}

In this study, 2228 T2DM patients were included from different provinces and different age groups, with $44.6 \%$ aged $45-55$ years. Approximately $40 \%$ had diabetes for more than five years. The patients were treated with different types of antidiabetic therapy ( $40.1 \%$ insulin). Their diabetes care was mainly provided by governmental primary health care centers ( $71.6 \%$ ). Only $48.3 \%$ were confident about their diabetes management, and 38.6\% were aware of their diabetes care. Many (83.4\%) patients managed their lifestyle, and $27.6 \%$ preferred self-medication. Only $17.7 \%$ were unsatisfied with their care provided by physicians, $28.9 \%$ had no physician consultation within the last 12 months, and $36.9 \%$ used to see their physician every three months (Table 1). 


\section{Cureus}

\begin{tabular}{|c|c|c|c|}
\hline \multicolumn{2}{|l|}{ Characteristics } & $\mathbf{N}$ & $\%$ \\
\hline \multirow{5}{*}{ Saudi Arabia residency } & North & 314 & $14.1 \%$ \\
\hline & West & 352 & $15.8 \%$ \\
\hline & East & 486 & $21.8 \%$ \\
\hline & Central & 534 & $24.0 \%$ \\
\hline & South & 542 & $24.3 \%$ \\
\hline \multirow{4}{*}{ Age (years) } & $18-25$ & 35 & $1.6 \%$ \\
\hline & $25-35$ & 598 & $26.8 \%$ \\
\hline & $35-45$ & 601 & $27.0 \%$ \\
\hline & $45-55$ & 994 & $44.6 \%$ \\
\hline \multirow{5}{*}{ Duration of diabetes } & 1 year & 564 & $25.3 \%$ \\
\hline & 2 years & 281 & $12.6 \%$ \\
\hline & 3 years & 253 & $11.4 \%$ \\
\hline & 4 years & 251 & $11.3 \%$ \\
\hline & $\geq 5$ years & 879 & $39.5 \%$ \\
\hline Smoking & & 659 & $29.6 \%$ \\
\hline \multirow{4}{*}{ Treatment of diabetes } & Lifestyle & 468 & $21.0 \%$ \\
\hline & Low carb & 180 & $8.1 \%$ \\
\hline & Oral drugs & 687 & $30.8 \%$ \\
\hline & Insulin & 893 & $40.1 \%$ \\
\hline \multirow{2}{*}{ Site of diabetes care } & Private & 632 & $28.4 \%$ \\
\hline & Governmental & 1596 & $71.6 \%$ \\
\hline \multirow{3}{*}{ Frequency of follow-up } & Monthly & 446 & $20.0 \%$ \\
\hline & Every 3 months & 823 & $36.9 \%$ \\
\hline & Every 6 months & 959 & $43.0 \%$ \\
\hline \multirow{2}{*}{ Physician consultation within 12 months } & No & 643 & $28.9 \%$ \\
\hline & Yes & 1585 & $71.1 \%$ \\
\hline \multirow{3}{*}{ Satisfaction with physicians' care } & Not satisfied & 395 & $17.7 \%$ \\
\hline & Satisfied & 1340 & $60.1 \%$ \\
\hline & Very Satisfied & 493 & $22.1 \%$ \\
\hline \multirow{2}{*}{ Lifestyle management } & No & 370 & $16.6 \%$ \\
\hline & Yes & 1858 & $83.4 \%$ \\
\hline Self-medication & & 615 & $27.6 \%$ \\
\hline \multirow{2}{*}{ Confidence in diabetes management } & Concerned & 1152 & $51.7 \%$ \\
\hline & Confident & 1076 & $48.3 \%$ \\
\hline \multirow{2}{*}{ Awareness of diabetes care } & Unaware & 1367 & $61.4 \%$ \\
\hline & Aware & 861 & $38.6 \%$ \\
\hline
\end{tabular}

TABLE 1: Characteristics of the participating diabetic patients $(n=2,228)$ 


\section{Cureus}

The participants' DSM score is shown in Figure 2, where they had an average score of 5.2/10. They had low scores in dietary (4.2/10), physical activity (4.2/10), and glucose management (4.7/10), with high scores in health care use (6.7/10).

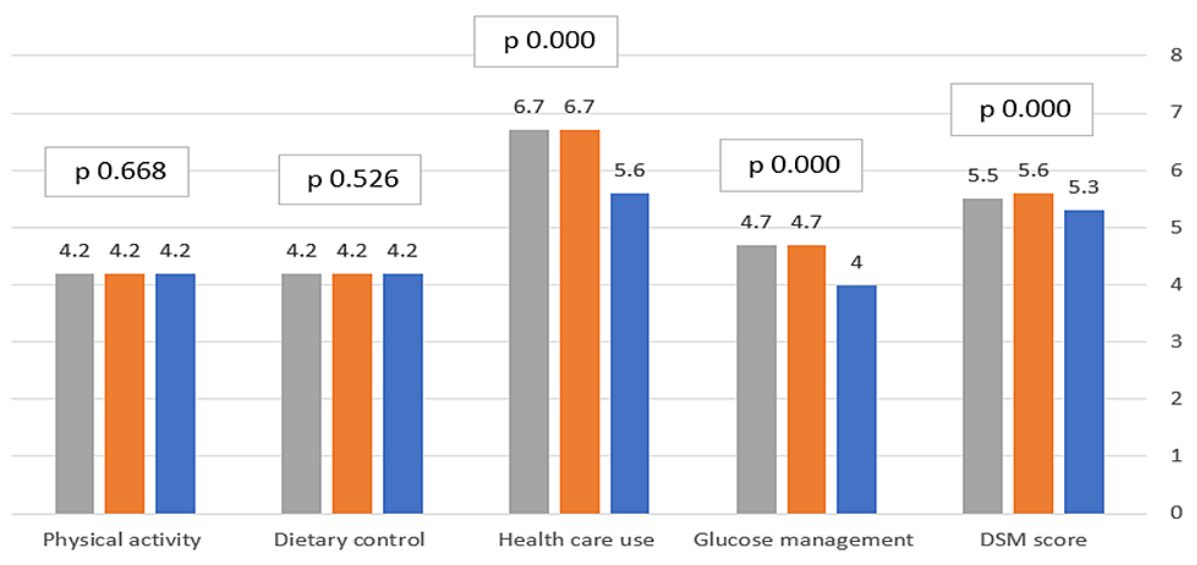

a-health dependent group घe-health independent group $\quad$ all participants

FIGURE 2: Diabetes self-management care items $(n=2,228)$

Diabetics with eHealth dependency showed significantly lower total DSM (5.6 vs. $5.3 ; \mathrm{p}=0.000$ ), especially because of the significantly lower health care use ( $6.7 \mathrm{vs.} 5.6 \% ; \mathrm{p}=0.000$ ) and glucose management ( $4.7 \mathrm{vs}$. $4.0 ; \mathrm{p}=0.000$ ) compared to the independent group (Figure 2).

Table 2 shows that $78.1 \%$ of the participants were using eHealth, $60.1 \%$ used it regularly, and $10.2 \%$ daily. The most frequent methods were Google (55.7\%) followed by social media (12.9\%), and the most frequent site was the SEHA app (54.7\%). Most diabetics (82\%) were like to discuss the eHealth information with their physicians; while only $34.5 \%$ had an online access to their physicians. Among diabetics, many found that eHealth information is of good (25.3\%) or even excellent (41.8\%) quality. Several of them were satisfied (16.5\%) or very satisfied (55.1\%) with eHealth to the extent of replacing physician consultation (44.2\%), and they were considered the eHealth-dependent group (Table 2). 


\section{Cureus}

\begin{tabular}{|c|c|c|c|}
\hline & & $\mathbf{N}$ & $\%$ \\
\hline \multirow{3}{*}{ Use of eHealth } & No & 488 & $21.9 \%$ \\
\hline & Yes & 1339 & $60.1 \%$ \\
\hline & Occasionally & 401 & $18.0 \%$ \\
\hline \multirow{4}{*}{ eHealth sources } & Google & 1242 & $55.7 \%$ \\
\hline & Social Media & 288 & $12.9 \%$ \\
\hline & YouTube & 194 & $8.7 \%$ \\
\hline & All & 21 & $0.9 \%$ \\
\hline \multirow{3}{*}{ eHealth use frequency } & Once/month & 1263 & $56.7 \%$ \\
\hline & Once/week & 238 & $10.7 \%$ \\
\hline & Daily & 227 & $10.2 \%$ \\
\hline \multirow{3}{*}{ Main eHealth portals } & SEHA app & 1218 & $54.7 \%$ \\
\hline & SEHA web & 233 & $10.5 \%$ \\
\hline & WHO web & 157 & $7.0 \%$ \\
\hline \multirow{3}{*}{ eHealth quality } & Fair & 152 & $6.8 \%$ \\
\hline & Good & 931 & $41.8 \%$ \\
\hline & Excellent & 665 & $29.8 \%$ \\
\hline \multirow{2}{*}{ eHealth information discussed with physician } & No & 401 & $18.0 \%$ \\
\hline & Yes & 1827 & $82.0 \%$ \\
\hline \multirow{2}{*}{ Online communication with physician office } & No & 1459 & $65.5 \%$ \\
\hline & Yes & 169 & $34.5 \%$ \\
\hline \multirow{4}{*}{ Online communication with hospital } & Appointment & 341 & $15.3 \%$ \\
\hline & Prescription renewal & 117 & $5.3 \%$ \\
\hline & Consultation & 203 & $9.1 \%$ \\
\hline & Medical report & 115 & $5.2 \%$ \\
\hline \multirow{4}{*}{ Satisfaction with eHealth } & Not & 480 & $21.5 \%$ \\
\hline & Borderline & 152 & $6.8 \%$ \\
\hline & Satisfied & 368 & $16.5 \%$ \\
\hline & Very satisfied & 1228 & $55.1 \%$ \\
\hline \multirow{2}{*}{ eHealth information is enough to replace physician visits } & eHealth independent group & 1243 & $55.8 \%$ \\
\hline & eHealth-dependent group & 985 & $44.2 \%$ \\
\hline
\end{tabular}

TABLE 2: Frequency of using eHealth among the participating diabetic patients $(n=2,228)$

There are many factors underlying eHealth dependency, as shown in Table 3. Predictors were the region of residency (OR: 0.911; 95\% CI: 0.852-0.974; $\mathrm{p}=0.006$ ), smoking (OR: 0.765; 95\% CI: 0.626-0.935; $\mathrm{p}=0.009$ ), physician visit within 12 months (OR: 1.305; 95\% CI: 1.061-1.605; p 0.012), frequency of care visits (OR: 1.136; $95 \%$ CI: $1.006-1.283 ; \mathrm{p}=0.040$ ), awareness of diabetes care (OR: $0.720 ; 95 \%$ CI: $0.597-0.869 ; \mathrm{p}=$ 0.001 ), online access to physicians (OR: $0.573 ; 95 \%$ CI: 0.473-0.694; $p=0.000$ ), discussion of eHealth information with physicians (OR: 0.648; $95 \%$ CI: 0.506-0.830; $p=0.001$ ), self-medication (OR: $0.459 ; 95 \%$ CI: $0.370-0.569 ; \mathrm{p}=0.000$ ), concern about treatment (OR: 0.635 ; 95\% CI: $0.513-0.787 ; \mathrm{p}=0.000$ ), DSM (OR: 1.022; 95\% CI: $1.006-1.039 ; \mathrm{p}=0.007$ ), and eHealth satisfaction (OR: 0.889; 95\% CI: 0.822-0.962; $\mathrm{p}=0.003$ ). 


\begin{tabular}{|c|c|c|c|c|}
\hline & \multirow{2}{*}{ OR } & \multicolumn{2}{|l|}{$95 \% \mathrm{Cl}$} & \multirow{2}{*}{ p-Value } \\
\hline & & Lower & Upper & \\
\hline Region of residence & 0.911 & 0.852 & 0.974 & $0.006^{*}$ \\
\hline Diabetes duration & 1.007 & 0.944 & 1.074 & 0.828 \\
\hline Age & 1.011 & 0.901 & 1.134 & 0.855 \\
\hline Smoking & 0.765 & 0.626 & 0.935 & $0.009^{\star}$ \\
\hline Anti-diabetics & 1.023 & 0.939 & 1.113 & 0.606 \\
\hline Lifestyle modification & 0.862 & 0.668 & 1.111 & 0.251 \\
\hline Visit physician within 12 months & 1.305 & 1.061 & 1.605 & $0.012^{*}$ \\
\hline Site of consultation & 0.972 & 0.793 & 1.191 & 0.784 \\
\hline Frequency of visits & 1.136 & 1.006 & 1.283 & $0.040^{*}$ \\
\hline Awareness of diabetes care & 0.720 & 0.597 & 0.869 & $0.001^{\star}$ \\
\hline Online access to physicians & 0.573 & 0.473 & 0.694 & $0.000^{*}$ \\
\hline Discussion of eHealth information & 0.648 & 0.506 & 0.830 & $0.001^{\star}$ \\
\hline Satisfaction with physician diabetes care & 0.897 & 0.775 & 1.039 & 0.147 \\
\hline Self-medications & 0.459 & 0.370 & 0.569 & $0.000^{*}$ \\
\hline Concern about diagnosis & 0.906 & 0.730 & 1.125 & 0.371 \\
\hline Concern about treatment & 0.635 & 0.513 & 0.787 & $0.000^{\star}$ \\
\hline eHealth satisfaction & 0.889 & 0.822 & 0.962 & $0.003^{*}$ \\
\hline Diabetes self-management & 1.022 & 1.006 & 1.039 & $0.007^{*}$ \\
\hline
\end{tabular}

\section{TABLE 3: Factors underlying eHealth dependency among the study participants}

*Statistically significant $\mathrm{p}$-value $\leq 0.05$.

\section{Discussion}

This study shows that approximately three-quarters of T2DM patients use eHealth information, with $44.2 \%$ of them depending on information gained online to the extent of replacing the role of physicians. The primary sources of eHealth were Google and social media, especially from the "SEHA" application. The major factors associated with eHealth dependency were physician visits, discussion, and online communication and satisfaction with eHealth quality. Other factors included patient concerns, self-medication, and management. Unfortunately, the eHealth-dependent patients showed significantly lower DSM scores, especially with regard to the use of health care services and glucose management compared to the other independent groups.

A large number of eHealth users in this study is much higher than that reported by another Saudi study in Riyadh, where only $27.9 \%$ of the study participants were seeking diabetic information online. However, the results of this study were in parallel with the recent overall increase in Internet usage in Saudi Arabia [13] and other countries [21].

A substantial number of patients considered eHealth as a substitute for doctors' visits. Similarly, others reported that the use of eHealth may postpone or replace medical consultations [22] in up to $30 \%$ of patients [23]. This trend is seen well in young patients [24], probably because of their ability to use electronic options. However, this trend was not observed in the present study. There are many possible interactions between the use of eHealth and the health care services provided by the diabetes care team. The use of eHealth might be linked to a lower number of regular physician visits [13], as in our patients. On the contrary, information gained from the Internet might influence patients to seek medical advice, help them to discuss many important issues during their visit, or guide them after the visit for more information on emerging topics [25]. 
Lower use of health care services with lower glucose management was reported in our study among the eHealth-dependent group. These findings are inconsistent with another study that reported that people in poor health are more likely to seek disease-related information online and use health care services to a larger extent [26]. eHealth use is supposed to be a fundamental part of DSM by providing easily accessible patient education with a consequent reduction in diabetes education costs and burden [27].

Our results suggest that despite the increasing consumption of eHealth among diabetics, they still need to discuss their concerns about their disease with their physicians. Moreover, the dependency on eHealth in this study was linked to the DM patients' Internet satisfaction but not to their satisfaction with the services provided by the health care professionals. This could reflect continued trust in the health system. These results are concurrent with those of previous reports [28]. Therefore, health care providers need to maintain continuity of and access to remote care and consultation with their patients. This will open the door to the use of telemedicine options in diabetes care. Telemedicine has evolved over the last decades to improve the accessibility and quality of care among patients and health care providers and to solve health care access challenges, especially during crises, such as during the COVID-19 pandemic.

The main limitation of this study is the use of a cross-sectional study design, which, together with a voluntary response sampling technique, will limit the generalization of its results to all Saudi T2DM patients. Moreover, the arbitrary labeling of the eHealth-dependent group based on the answer to one question is another limitation of this study.

\section{Conclusions}

Most Saudi T2DM patients with average DSM use different eHealth resources and are satisfied with their quality. The dependency of eHealth is significantly associated with lower DSM, especially for health care use and glucose monitoring management, a finding that could affect patient outcomes. Still, patients need to communicate with their physicians in person, who should have different options for remote consultation, such as telemedicine, to support their patients.

\section{Additional Information \\ Disclosures}

Human subjects: Consent was obtained or waived by all participants in this study. Ibn Sina National College (ISNC) Research and Ethics Committee issued approval H-08-24102019. Animal subjects: All authors have confirmed that this study did not involve animal subjects or tissue. Conflicts of interest: In compliance with the ICMJE uniform disclosure form, all authors declare the following: Payment/services info: All authors have declared that no financial support was received from any organization for the submitted work. Financial relationships: All authors have declared that they have no financial relationships at present or within the previous three years with any organizations that might have an interest in the submitted work. Other relationships: All authors have declared that there are no other relationships or activities that could appear to have influenced the submitted work.

\section{References}

1. American Diabetes Association: Introduction: standards of medical care in diabetes-2021 . Diabetes Care. 2021, 44:1-2. 10.2337/dc21-Sint

2. Powers MA, Bardsley J, Cypress M, et al.: Diabetes self-management education and support in type 2 diabetes: a joint position statement of the American Diabetes Association, the American Association of Diabetes Educators, and the Academy of Nutrition and Dietetics. Diabetes Educ. 2017, 43:40-53. $10.1177 / 0145721715588904$

3. eHealth. (2018). Accessed: September 1, 2020: https://www.who.int/ehealth/about/en/.

4. Pagliari C, Sloan D, Gregor P, et al.: What is eHealth (4): a scoping exercise to map the field . J Med Internet Res. 2005, 7:9. 10.2196/jmir.7.1.e9

5. Hansen A, Claudi T, Årsand E: Use of electronic health and its impact on doctor-visiting decisions among people with diabetes: cross-sectional study. J Med Internet Res. 2019, 21:13678. 10.2196/13678

6. Hansen AH, Bradway M, Broz J, Claudi T, Henriksen Ø, Wangberg SC, Årsand E: The use of eHealth and provider-based health services by patients with diabetes mellitus: protocol for a cross-sectional study. JMIR Res Protoc. 2016, 5:207. 10.2196/resprot.6529

7. Madanian S, Norris T, Parry D: Disaster eHealth: scoping review. J Med Internet Res. 2020, 28:18310. $10.2196 \% 2 \mathrm{~F} 18310$

8. Weymann N, Dirmaier J, von Wolff A, Kriston L, Härter M: Effectiveness of a web-based tailored interactive health communication application for patients with type 2 diabetes or chronic low back pain: randomized controlled trial. J Med Internet Res. 2015, 17:53. 10.2196/jmir.3904

9. Moreau M, Gagnon M, Boudreau F: Development of a fully automated, web-based, tailored intervention promoting regular physical activity among insufficiently active adults with type 2 diabetes: integrating the I-change model, self-determination theory, and motivational interviewing components. JMIR Res Protoc. 2015, 4:25. 10.2196/resprot.4099

10. Glasgow RE, Kurz D, King D, et al.: Twelve-month outcomes of an Internet-based diabetes self-management support program. Patient Educ Couns. 2012, 87:81-92. 10.1016/j.pec.2011.07.024

11. Hansen AH, Broz J, Claudi T, Årsand E: Relations between the use of electronic health and the use of general 
practitioner and somatic specialist visits in patients with type 1 diabetes: cross-sectional study. J Med Internet Res. 2018, 7:11322. 10.2196/11322

12. de Boer MJ, Versteegen GJ, van Wijhe M: Patients' use of the Internet for pain-related medical information . Patient Educ Couns. 2007, 68:86-97. 10.1016/j.pec.2007.05.012

13. AlGhamdi KM, Moussa NA: Internet use by the public to search for health-related information . Int J Med Inform. 2012, 81:363-373. 10.1016/j.ijmedinf.2011.12.004

14. Ferguson MO, Long JA, Zhu J, Small DS, Lawson B, Glick HA, Schapira MM: Low health literacy predicts misperceptions of diabetes control in patients with persistently elevated A1C. Diabetes Educ. 2015, 41:309319. 10.1177/0145721715572446

15. Hilliard ME, Sparling KM, Hitchcock J, Oser TK, Hood KK: The emerging diabetes online community. Curr Diabetes Rev. 2015, 11:261-272.

16. IDF Diabetes Atlas. (2017). Accessed: September 1, 2020: http://fmdiabetes.org/wpcontent/uploads/2018/03/IDF-2017.pdf.

17. Jamal A, Khan S, AlHumud A, et al.: Association of online health information-seeking behavior and self-care activities among type 2 diabetic patients in Saudi Arabia. J Med Internet Res. 2015, 17:196. 10.2196/jmir.4312

18. Schmitt, A, Gahr A, Hermanns N, Kulzer B, Huber J, Haak T: The diabetes self-management questionnaire (DSMQ): development and evaluation of an instrument to assess diabetes self-care activities associated with glycaemic control. Health Qual Life Outcomes. 2013, 11:138. 10.1186/1477-7525-11-138

19. Bradley C: Translation of questionnaires for use in different languages and cultures . Handbook of Psychology and Diabetes: A Guide to Psychological Measurement in Diabetes Research and Practice. Harwood Academic Publishers, Chur, Switzerland; 1994. 43-55.

20. World Medical Association Declaration of Helsinki: ethical principles for medical research involving human subjects. (2008). Accessed: January 1, 2021: https://www.wma.net/policies-post/wma-declaration-ofhelsinki-ethical-principles-for-medical-research-involving-human...

21. Siliquini R, Ceruti M, Lovato E, et al.: Surfing the internet for health information: an italian survey on use and population choices. BMC Med Inform Decis Mak. 2011, 11:21. 10.1186/1472-6947-11-21

22. Akerkar SK, Kanitkar M, Bichile LS: Use of the Internet as a resource of health information by patients: a clinic-based study in the Indian population. J Postgrad Med. 2005, 51:116-118.

23. Beck F, Richard JB, Nguyen-Thanh V, Montagni I, Parizot I, Renahy E: Use of the Internet as a health information resource among French young adults: results from a nationally representative survey. J Med Internet Res. 2014, 16:128. 10.2196/jmir.2934

24. Bauer AM, Rue T, Keppel GA, Cole AM, Baldwin LM, Katon W: Use of mobile health (mHealth) tools by primary care patients in the WWAMI region Practice and Research Network (WPRN). J Am Board Fam Med. 2014, 27:780-788. 10.3122/jabfm.2014.06.140108

25. McMullan M: Patients using the Internet to obtain health information: how this affects the patient-health professional relationship. Patient Educ Couns. 2006, 63:24-28. 10.1016/j.pec.2005.10.006

26. Nölke L, Mensing M, Krämer A, Hornberg C: Sociodemographic and health-(care-)related characteristics of online health information seekers: a cross-sectional German study. BMC Public Health. 2015, 15:31. 10.1186/s12889-015-1423-0

27. Hickson R, Talbert J, Thornbury WC, Perin NR, Goodin AJ: Online medical care: the current state of "eVisits" in acute primary care delivery. Telemed J E Health. 2015, 21:90-96. 10.1089/tmj.2014.0022

28. Thackeray R, Crookston B, West J: Correlates of health-related social media use among adults. J Med Internet Res. 2013, 15:21. 10.2196/jmir.2297 